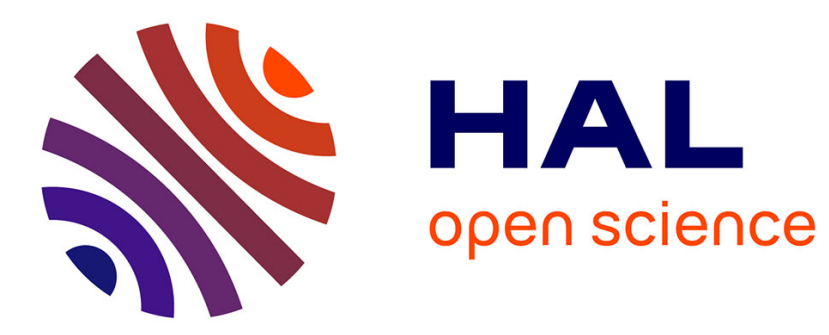

\title{
Improving sensor noise analysis for CT-Scanner identification
}

\author{
Anas Kharboutly, William Puech, Gérard Subsol, Denis Hoa
}

\section{To cite this version:}

Anas Kharboutly, William Puech, Gérard Subsol, Denis Hoa. Improving sensor noise analysis for CTScanner identification. EUSIPCO: European Signal Processing Conference, Aug 2015, Nice, France. pp.2411-2415, 10.1109/EUSIPCO.2015.7362817 . lirmm-01379558

\section{HAL Id: lirmm-01379558 https://hal-lirmm.ccsd.cnrs.fr/lirmm-01379558}

Submitted on 27 Mar 2019

HAL is a multi-disciplinary open access archive for the deposit and dissemination of scientific research documents, whether they are published or not. The documents may come from teaching and research institutions in France or abroad, or from public or private research centers.
L'archive ouverte pluridisciplinaire HAL, est destinée au dépôt et à la diffusion de documents scientifiques de niveau recherche, publiés ou non, émanant des établissements d'enseignement et de recherche français ou étrangers, des laboratoires publics ou privés. 


\title{
IMPROVING SENSOR NOISE ANALYSIS FOR CT-SCANNER IDENTIFICATION
}

\author{
Anas Kharboutly*, William Puech*, Gérard Subsol ${ }^{*}$, Denis Hoa ${ }^{\dagger}$ \\ * ICAR Research Team, LIRMM, University of Montpellier/CNRS, Montpellier, France \\ ${ }^{\dagger}$ IMAIOS, MIBI, Montpellier, France
}

\begin{abstract}
CT-Scanner devices produce three-dimensional images of the internal structure of the body. In this paper, we propose a method that is based on the analysis of sensor noise to identify the CT-Scanner device. For each CT-scanner we built a reference pattern noise and a correlation map from its slices. Finally, we can correlate any test slice with the reference pattern noise of each device according to its correlation map. This correlation map gives a weighting for each pixel regarding its position in the reference pattern noise. We used a waveletbased Wiener filter and an edge detection method to extract the noise from a slice. Experiments were applied on three CT-Scanners with 40 3D images, including 3600 slices, and we demonstrate that we are able to identify each CT-Scanner separately.
\end{abstract}

Index Terms - Digital forensics, medical image forensics, authentication, device identification, noise pattern, sensor noise, denoise filtering, edge detection, wavelet transformation.

\section{INTRODUCTION}

Medical imaging is considered an important part of the medical world, this refers to the process and technique used to obtain an internal visualization of the human body. Computed tomography [1] or what is called CT-Scanners provide high quality images. These images are produced by integrating a series of X-ray views from many different angles to build cross-sectional images. Unlike other imaging techniques, CTScanners provide detailed images of many types of tissue, including bone, lung tissue, soft tissue and blood vessels. It is used to build an internal representation of almost all body parts.

Medical images are stored in general as DICOM files [2]. A DICOM file is consisted of two parts, meta-data and the image content. Meta-data is a readable file, it contains all the information about the acquisition device, the acquisition system and the image parameters. Meta-data files may be modified, become corrupted or even lost. Consequently, it is very important to be able to retrieve as much information as possible from the image part only. In particular, in the absence of meta-data, or if they are not authenticated, we would like to identify the CT-Scanner device from raw images and this is exactly the objective of image forensics [3]. Image forensics is an important research area, its basic concern is to authenticate the images by retrieving information about their source in case of unauthenticated device or image modification. These techniques are based on the images themselves without any prior information [4].

When we talk about image forensics, two basic problems are raised: forgery tracing and device identification. In forgery tracing, much work already exists on digital photography [5], but on the medical side, very few research has been conducted. In [6] the authors present research about the digital blind forensics, they propose a method for detecting the forged images. Regarding the device identification, in [7-9] the authors propose a method for digital camera identification, it is based on the sensor noise analysis, they present a denoising method and build a Reference Pattern Noise, this RPN has served as a unique fingerprint for each camera, this work was carried out on the digital images. But in the medical domain, very few works exist. In [10], the authors compared the noise characteristics between two CTScanners from different manufacturers, then they linked the reconstruction core between two devices from Siemens and General Electric. In [11], the authors proposed a method for device identification, but only for the primitive $2 \mathrm{D}$ images of $\mathrm{X}$-ray radiography.

Looking in greater detail at CT-Scanner identification, we proposed in [12] a first analysis of this problem. We used a wavelet-based denoising algorithm based on the method proposed in [7] to extract the noise in CT slices. But we had some difficulties, as current CT images contain many anatomical structures which are contrasted with relation to each other and especially with relation to the background. This creates many edges and is not easy to distinguish these high frequency features from the ones created by the noise. In this paper, we improve the denoising algorithm by including a correlation map as suggested in [13] which takes into account the reliability of the noise computation from a given pixel of a CT slice. We include a mask that eliminates the edges and traces those are left with the noise in the high frequency range, we built a reference pattern noise for each device. A correlation map was also built for each device for better correlation computation. Then we are able to identify the CT-Scanner based on the correlation between the tested slices and the reference pattern noise, the correlation was basically completed using the correlation map, this correlation map decides the importance of the pixel position, it gives a weighting factor as a 
percentage for each pixel, this percentage was computed using the pixel position being used as an edge or not.

In Section 2, we describe our new version of the CTScanner identification method and we focus on the improvement of the denoising algorithm by using the correlation map. In Section 3, we present some experimental results in addition to some further discussions. In Section 4, we discuss some ideas for future research.

\section{THE PROPOSED METHOD}

For each device we built a reference pattern noise and a correlation map. To identify a specific CT-Scanner as the acquisition device of a tested slice, we compute the correlation between the noise component of this slice and the reference pattern noise of this device, this correlation is computed depending on the correlation map. The correlation value between the noise component of this tested slice and the reference pattern noise of the acquisition device should always be the highest. Fig.1 illustrates the method overview.

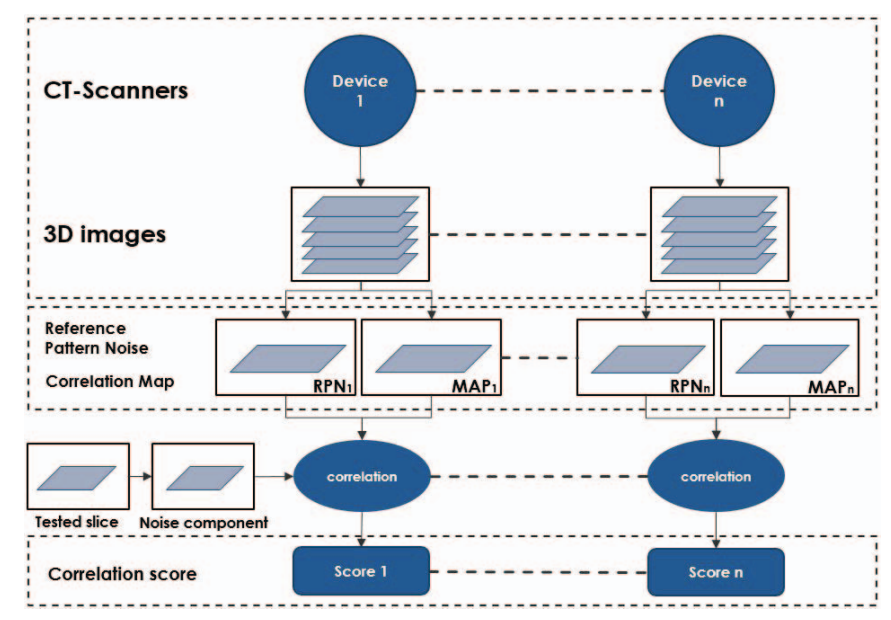

Fig. 1. Method overview 1.

In this section, we will present our proposed denoising method which is used to extract the noise component of each studied slice, how we can build both the reference pattern noise and the correlation map and finally, how do we compute the correlation between noise component of the tested slice and the reference pattern noise depending on the correlation map in order to decide the acquisition device.

\subsection{Denoising algorithm}

In the frequency domain, we applied a Wiener filter based wavelet transformation [14-16]. Basically, this algorithm is composed of two parts. First; local variance estimation of the wavelet components. Second; denoising of these components using Wiener filter (see a tutorial in [17]) as follows:

- Calculate four levels of wavelet decomposition of the original slice. In each level, take out the three high fre- quency sub-bands: horizontal, vertical and diagonal. For four levels of wavelet decomposition with three sub-bands in each level we have 12 sub-bands for each processed slice.

- For each wavelet sub-band, estimate the local variance. Four variances values are computed regarding four sizes of pixel area $\{35,7,9\}$, then the smallest one is the final estimated variance.

- Denoise each wavelet sub-band using Wiener filter:

$$
X_{d e n}(i, j)=X(i, j) \frac{\hat{\sigma}^{2}(i, j)}{\hat{\sigma}^{2}(i, j)+\sigma_{0}^{2}},
$$

where $X$ is the wavelet sub-band, $\hat{\sigma}^{2}(i, j)$ is the estimated variance of each pixel, $\sigma_{0}$ is an initial integer constant value that we tuned manually, $\sigma_{0} \in[1,6]$.

- Apply inverse wavelet transformation on the denoised wavelet sub-bands to extract the denoised component $F(s)$ of the original slice $s$.

\subsection{Noise extraction and correction}

Our basic concern is about noise, in order to extract the noise component, we subtract the denoised slice from the original one.

$$
n^{(i)}=s^{(i)}-F\left(s^{(i)}\right),
$$

where $n$ is the noise component, $s$ is the slice, $F()$ is the denoising function and $i$ is the slice number.

As a result of the subtraction operation we get the noise component, but in addition to the noise, there exist other traces or edges that remain in the noise slice. To deal with these kinds of traces, we apply a noise correction step. We apply an edge detection on the original image in order to build a mask of the edges that existed in the slice [18] as follow:

- Apply a blurring filter to remove the noise.

- Compute the gradient of each pixel.

- Compute the norm of the gradient for each pixel and create an image of the values.

- Finally, threshold the norm image to extract the maximum local values that represents the edge mask.

Finally, we applied this edge mask on the previous noise component computed from equation 2 , the result of the masked slice represents the pure noise component, this component does not contain any traces or edges as illustrated in Fig.2

In Fig.2, an example of a slice from a 3D image of phantom that were acquired by a Siemens device, where (a) is the original slice, (b) contains noise in addition to some traces and (c) contains noise only without any trace or edge.

\subsection{CT-Scanner reference pattern and correlation map}

To build the reference pattern noise, we selected a set of slices regarding the CT-Scanner device, we denoised these slices. 


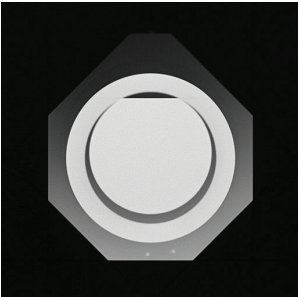

(a)

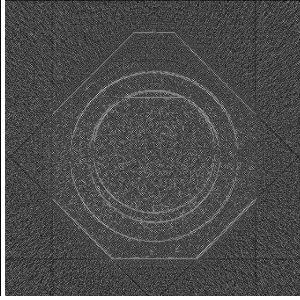

(b)

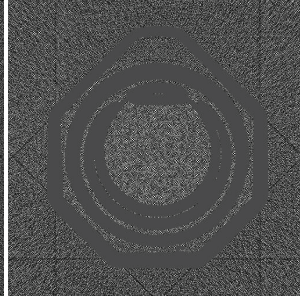

(c)
Fig. 2. (a) Example of a slice from CT-Scanner from Siemens, (b) Its noise component, (c) Its noise component without edges.

Then, we calculated the average of slices of the pure noise component, the average result represents the reference pattern noise RPN or what is called the device fingerprint:

$$
R P N=\frac{1}{N} \sum_{i=1}^{N} n^{(i)}
$$

where $R P N$ is the reference pattern noise, $N$ is the number of noise slices and $n$ is the noise component.

We are dealing with $3 \mathrm{D}$ volumes consisting of multiple slices, we applied an average operation according to ' $z$ ' axis to extract the $2 \mathrm{D}$ reference pattern noise. In some slices, we remove the traces that remain in the noise component, so according to the pixel position, in some slices, this pixel may have served as an edge and it has been removed. Consequently, this pixel position loses some information regarding the ' $Z$ ' directional axis, while another pixel position did not lose any information since it has not served as an edge. We built what we called a correlation map as suggested in [13]. The correlation map gives information about the importance of each pixel position.

Regarding the selected slices of the reference pattern noise, we built the correlation map, where the pixel value represent its frequency as an edge in these slices:

$$
\operatorname{map}(i, j)=\sum_{I \in R P N \text { slices }} \operatorname{mask}_{I}(i, j)
$$

where map is the edge frequencies and mask $k_{I}$ is the edge mask of $I$.

To convert the values of correlation map into percentage values, we inverted the map values and divided each pixel value by the maximum one, so each pixel value represents its importance regarding its frequency as an edge. The pixel with the highest edge frequency has a low importance and so it takes the value of 0 , while the pixel with the lowest edge frequency has a high importance and it takes the value 1:

$$
\operatorname{cor} r \_\operatorname{map}(i, j)=\frac{\operatorname{Inv}(\operatorname{map}(i, j))}{\max (\operatorname{map})},
$$

where corr_map is the final correlation map, Inv is the inversion factor and $\max$ is the maximum value of $\operatorname{map}$.

\subsection{Decision by correlation}

Looking at each device, we have a reference pattern noise and a correlation map. In order to test new slices to know from which device they were acquired, we compute the correlation between the reference pattern noise of each device and the noise component of each slice depending on the weighting factor of the correlation map. The tested slice is identified as acquired from a specific device when it has the highest correlation value with its reference pattern noise:

$$
\widetilde{R P N}(i, j)=R P N(i, j) \times \operatorname{map}(i, j)
$$

where $R P N$ is the reference pattern noise and map is the correlation map.

$$
\tilde{n}(i, j)=n(i, j) \times \operatorname{map}(i, j),
$$

where $n$ is the pure noise component and map is the correlation map. Finally, the correlation is:

$$
\operatorname{corr}\left(\tilde{n}_{(z)}, \widetilde{R P N}\right)=\frac{\left(\tilde{n}_{(z)}-\overline{\tilde{n}}_{(z)}\right) \cdot(\widetilde{R P N}-\overline{R P N})}{\left\|\tilde{n}_{(z)}-\overline{\tilde{n}}_{(z)}\right\|\|\widetilde{R P N}-\widetilde{\widetilde{R P N}}\|}
$$

where $z$ is the slice number, the correlation was applied on the unmasked pixels only.

\section{EXPERIMENTAL RESULTS}

We applied our experiments on 40 3D images of 3600 slices from three different CT-Scanners, 12 3D images of 1200 slices from the Siemens 1, 12 3D images of 1200 slices from the Siemens 2 and 16 3D images of 1200 slices from General Electric. These images have similar acquisition parameters (Beam energy: $(120,140) \mathrm{KV}$, Pitch value: $(0.5,1)$, Reconstruction: (soft, hard)) and the other parameters are illustrated in Table 1.

\begin{tabular}{|l|l|l|l|}
\hline & Siemens 1 & Siemens 2 & GE \\
\hline Content & phantom & phantom & phantom \\
\hline Nb of images & 12 & 12 & 16 \\
\hline Nb of slices & 1200 & 1200 & 1200 \\
\hline Size (pixels) & $512 \times 512$ & $512 \times 512$ & $512 \times 512$ \\
\hline Bits per pixel & 16 & 16 & 16 \\
\hline Slice thickness & $3 \mathrm{~mm}$ & $3 \mathrm{~mm}$ & $3 \mathrm{~mm}$ \\
\hline Pixel size & $1 \mathrm{~mm}$ & $1 \mathrm{~mm}$ & $1 \mathrm{~mm}$ \\
\hline Nb of slices of RPN & 200 & 200 & 200 \\
\hline Nb of tested slices & 1000 & 1000 & 1000 \\
\hline
\end{tabular}

Table 1. Characteristics of the experimental images.

200 slices were selected randomly from each device to build the reference pattern noise as illustrated in Fig. 3, the correlation map was built depending on the slices of the reference pattern noise of each device. To build the correlation map, 


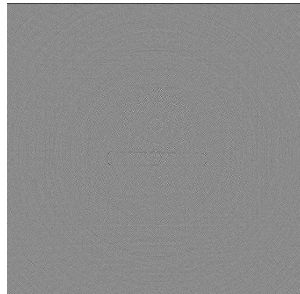

(a) RPN of S1

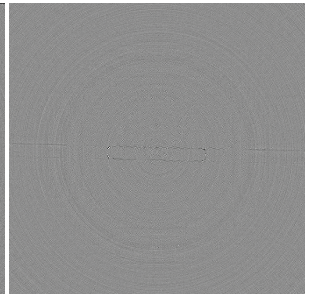

(b) RPN of S2

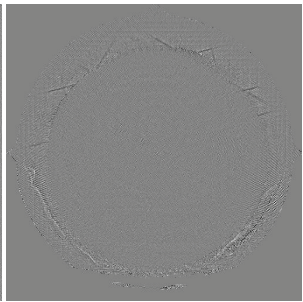

(c) RPN of GE
Fig. 3. The reference pattern noise of each device.

each pixel of the correlation map represents the frequency of the same pixel in all the device slices being served as an edge as illustrated in Fig. 4.

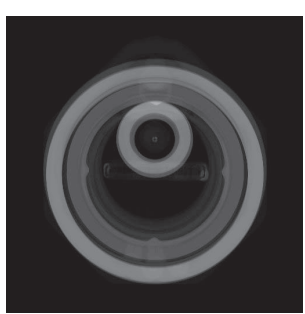

(a) Map of S1

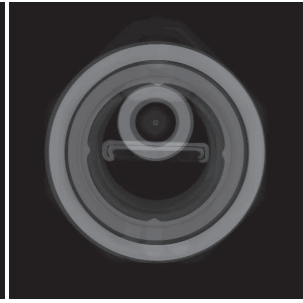

(b) Map of S2

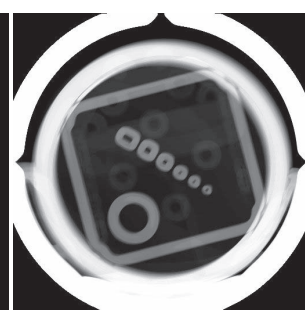

(c) Map of GE
Fig. 4. The correlation map of each device.

And the resulted reference pattern noise of each device according to equation 6 is illustrated in Fig. 5.

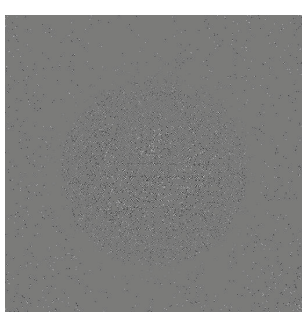

(a) RPN of S 1

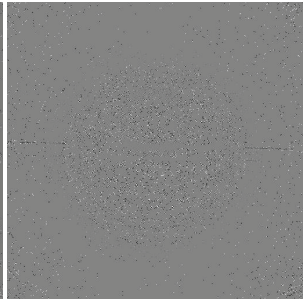

(b) RPN of S 2

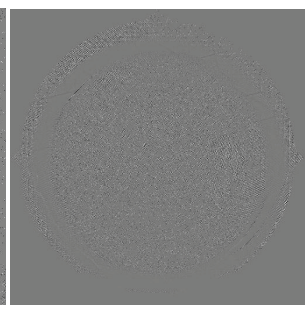

(c) RPN of GE
Fig. 5. The three RPNs according to (6).

Generally, the General Electric images are centered in a circle of diameter equal to the image hight or width, so we build a circle mask with a size equal to that of General Electric, in order to keep a common element among all the references. Then, we applied this mask on each reference pattern noise and tested slice. Finally, we calculated the correlation, between the noise component of the tested slices and the reference pattern noise of each device, the correlation is dependent on the correlation map as illustrated in Fig. $(6,7,8)$. In each plot, the ' $\mathrm{x}$ ' axis represents the tested slice number and the ' $y$ ' axis represents the correlation value. We could notice that the correlation values between the tested slices and the reference pattern noise of the same device are the highest. Table 2 illustrates the identification accuracy regarding each device:

\begin{tabular}{|l|l|l|l|}
\hline & Siemens 1 & Siemens 2 & GE \\
\hline Siemens 1 & $94,3 \%$ & $2,3 \%$ & $0 \%$ \\
\hline Siemens 2 & $2,6 \%$ & $95,2 \%$ & $0 \%$ \\
\hline GE & $3,1 \%$ & $2,5 \%$ & $100 \%$ \\
\hline
\end{tabular}

Table 2. Identification accuracy.

- 943 slices of Siemens 1 were classified correctly as acquired from Siemens 1, while 57 slices were not.

- 952 slices of Siemens 2 were classified correctly as acquired from Siemens 2, while 48 slices were not.

- 1000 slices of General Electric were classified correctly as acquired from GE.

\section{CONCLUSION AND FUTURE WORK}

In this paper, we proposed an improvement to the analysis of the CT-Scanner identification problem. As a method of medical image forensics, we were able to identify three different CT-Scanners from their original images.

For future research, we plan to work directly on 3D images, to study the influence of different acquisition parameters and we are going to study the influence of image compressing on our proposed method.

\section{REFERENCES}

[1] J. T. Bushberg and J. M. Boone, The essential physics of medical imaging, Lippincott Williams \& Wilkins, 2011.

[2] K. D. Toennies, Guide to Medical Image Analysis Methods and Algorithms, Advances in Computer Vision and Pattern Recognition. Springer, 2012.

[3] J. Redi, W. Taktak, and J. L. Dugelay, "Digital image forensics: a booklet for beginners," Multimedia Tools and Applications, vol. 51, no. 1, pp. 133-162, 2011.

[4] A. Piva, "An overview on image forensics," ISRN Signal Processing, vol. 2013, 2013.

[5] H. T. Sencar and N. Memon, Digital Image Forensics: There is More to a Picture Than Meets the Eye, Springer, 2013.

[6] H. Huang, G. Coatrieux, H. Shu, L. Luo, and C. Roux, "Blind integrity verification of medical images," IEEE Transactions on Information Technology in Biomedicine, vol. 16, no. 6, pp. 1122-1126, 2012.

[7] J. Lukas, J. Fridrich, and M. Goljan, "Digital camera identification from sensor pattern noise," IEEE Transactions on Information Forensics and Security, vol. 1, no. 2, pp. 205-214, 2006.

[8] J. Fridrich, "Digital image forensics," Signal Processing Magazine, IEEE, vol. 26, no. 2, pp. 26-37, 2009. 


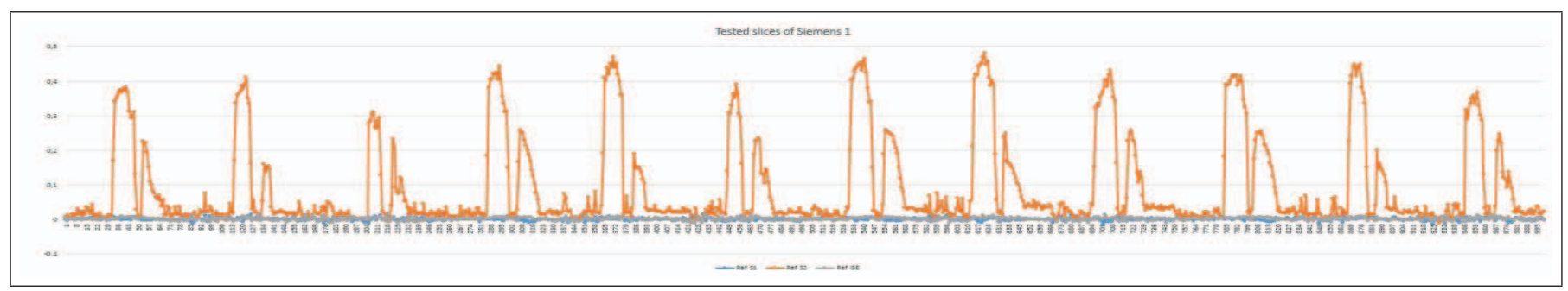

Fig. 6. Correlation between 1000 tested slices of Siemens 1 and the RPN of each device.

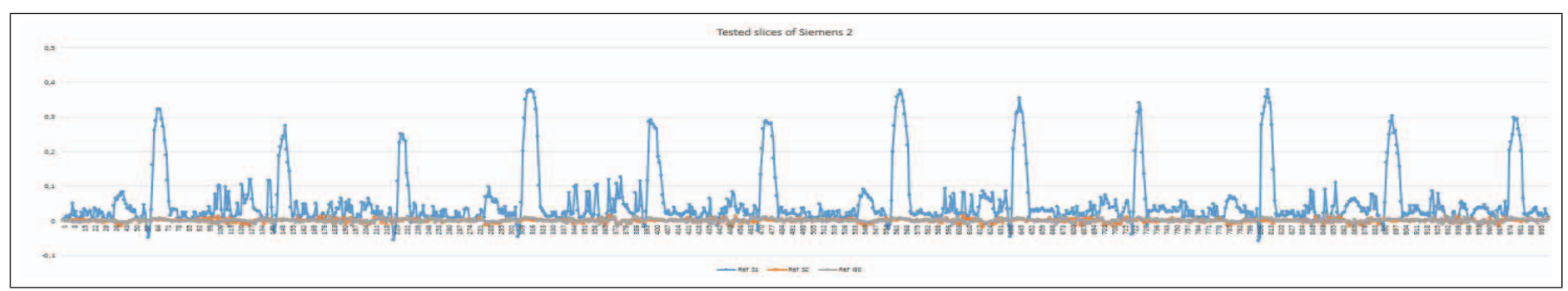

Fig. 7. Correlation between 1000 tested slices of Siemens 2 and the RPN of each device.

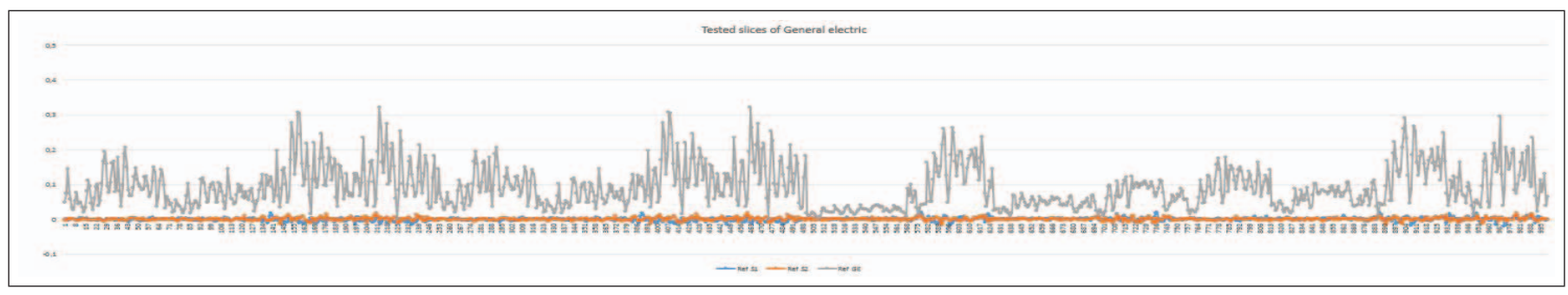

Fig. 8. Correlation between 1000 tested slices of GE and the RPN of each device.

[9] M. Chen, J. Fridrich, M. Goljan, and J. Lukás, "Determining image origin and integrity using sensor noise," Information Forensics and Security, IEEE Transactions on, vol. 3, no. 1, pp. 74-90, 2008.

[10] J. B. Solomon, O. Christianson, and E. Samei, "Quantitative comparison of noise texture across CT scanners from different manufacturers," Medical physics, vol. 39, no. 10, pp. 6048-55, October 2012.

[11] Y. Duan, G. Coatrieux, and H. Shu, "Identification of digital radiography image source based on digital radiography pattern noise recognition," in Image Processing (ICIP), 2014 IEEE International Conference on. IEEE, 2014, pp. 5372-5376.

[12] A. Kharboutly, W. Puech, G. Subsol, and D. Hoa, "Ctscanner identification based on sensor noise analysis," in Visual Information Processing (EUVIP), 2014 5th European Workshop on. IEEE, 2014, pp. 1-5.

[13] L.-H. Chan, N.-F. Law, and W.-C. Siu, "A confidence map and pixel-based weighted correlation for prnubased camera identification," Digital Investigation, vol. 10, no. 3, pp. $215-225,2013$.
[14] M.K. Mihçak, I Kozintsev, and K. Ramchandran, "Spatially adaptive statistical modeling of wavelet image coefficients and its application to denoising," in Acoustics, Speech, and Signal Processing, 1999. Proceedings., 1999 IEEE International Conference on, Mar 1999, vol. 6, pp. 3253-3256.

[15] E. Jerhotová, A. Procházka, and J. Švihlík, Biomedical image volumes denoising via the wavelet transform, INTECH Open Access Publisher, 2011.

[16] P. Gravel, G. Beaudoin, and J. A. De Guise, "A method for modeling noise in medical images," Medical Imaging, IEEE Transactions on, vol. 23, no. 10, pp. 12211232, 2004.

[17] N. Jacob and A. Martin, "Image denoising in the wavelet domain using Wiener filtering," Unpublished course project, 2004, [Online], Project Report, Available: http://homepages.cae.wisc.edu/ ece533/project/ f04/jacob_martin.pdf.

[18] M. Nitzberg, D. Mumford, and T. Shiota, Filtering, Segmentation, and Depth, Lecture notes in computer science. Springer-Verlag, 1993. 